\title{
Canted magnetization texture in ferromagnetic tunnel junctions
}

\author{
Igor Kuzmenko and Vladimir Fal'ko \\ Department of Physics, Lancaster University, Lancaster LA1 4YB, United Kingdom \\ (Received 18 August 2008; revised manuscript received 27 October 2008; published 20 November 2008)

\begin{abstract}
We study the formation of inhomogeneous magnetization texture in the vicinity of a tunnel junction between two metallic ferromagnets nominally in the antiparallel configuration and its influence on the magnetoresistance of such a device. Such a texture, dependent on the magnetization rigidity and crystalline anisotropy energy in the ferromagnet, develops when the ferromagnetic coupling across the tunnel barrier increases above a critical value.
\end{abstract}

DOI: 10.1103/PhysRevB.78.184425

PACS number(s): 72.25.Mk, 75.47.De, 75.60.Ch

Spin-polarized transport in ferromagnetic tunnel junction is a subject of intense theoretical and experimental studies. ${ }^{1,2}$ Most of these studies address the investigation of the tunneling magnetoresistance ${ }^{3-5}$ (MR) and giant MR (Refs. 6-10) effects, which consist of a switch from lower to higher conductivity when the polarization of leads in a MR device changes from an antiparallel to a parallel configuration. The MR effect is the result of a difference between rates of tunneling of electrons in the majority and minority bands on the opposite sides of a junction, and it is strongest when magnetization switching by an external magnetic field changes from an ideally antiparallel magnetization state on the two sides of a tunnel junction to a parallel one. ${ }^{11-14}$ Any deviations in the magnetization near the interface (where the tunneling characteristics of the device are formed) from perfectly parallel or antiparallel orientation would reduce the size of the effect.

In this paper, we investigate the possibility of the formation of inhomogeneous magnetization texture in the vicinity of a highly transparent tunnel junction caused by ferromagnetic coupling of magnetic moments on opposite sides of the junction. Such a coupling would be stronger in devices with more transparent barriers, that is, with a high tunneling conductance. We find that a canted magnetization state can form if the ferromagnetic tunneling coupling, $t^{\prime}$, exceeds some critical value $t_{0}$ determined by the interplay between crystalline anisotropy and magnetization rigidity in the ferromagnet. A tunnel junction with $t^{\prime}<t_{0}$ can be viewed as an atomically sharp magnetic domain wall, whereas the increase in the junction transparency above $t_{0}$ gradually transforms it into a broad texture typical for a domain wall in a bulk ferromagnetic material. For $t^{\prime}>t_{0}$, we study the evolution of the texture upon application of an external magnetic field and we construct a parametric diagram for distinct magnetization regimes. As an example, we consider a device consisting of a tunnel junction between two easy-axis ferromagnets magnetically biased at the ends, as illustrated in Figs. 1(a) and 1(b). When the magnetic field exceeds some critical value, $B_{0}$, the domain wall is pushed away toward the magnetically biased end of the ferromagnetic metal. When a magnetic field is swept back and its sign changes, the domain wall returns back to the tunnel junction. The resulting hysteresis in the magnetization state of the device leads to a hysteresis loop in its MR, which we analyze, taking into account the formation of the texture near the tunnel junction.

A quasi-one-dimensional (quasi-1D) magnetization tex- ture, $\mathbf{S}(l)$, near the tunnel junction, which changes slowly on the scale of the lattice constant $a$ can be described using the energy functional

$$
\begin{aligned}
E= & \frac{J}{2}\left(\int_{-L}^{-0}+\int_{+0}^{L}\right) d l\left[\partial_{l} \mathbf{S}\right]^{2}-\frac{w^{2}}{a^{3}} \int_{-L}^{L} d l\left\{\xi_{1}\left[S^{z}\right]^{2}+\xi_{2}\left[S^{y}\right]^{2}\right\} \\
& -\frac{\mu B w^{2}}{a^{3}} \int_{-L}^{L} d l S^{z}+\frac{w^{3}}{2 a^{3}} \int_{-L}^{L} \frac{d l d l^{\prime}}{w^{2}} V\left(l-l^{\prime}\right) \\
& \times\left\{S^{x}(l) S^{x}\left(l^{\prime}\right)+S^{y}(l) S^{y}\left(l^{\prime}\right)-2 S^{z}(l) S^{z}\left(l^{\prime}\right)\right\}+E^{\prime} .
\end{aligned}
$$

Here, the first term describes the exchange interaction between the neighboring atoms giving rise to the magnetization rigidity $J \sim t w^{2} / a$ (where $t$ is the exchange coupling and $w^{2}$ is the cross-sectional area of the ferromagnet). The second

(a)

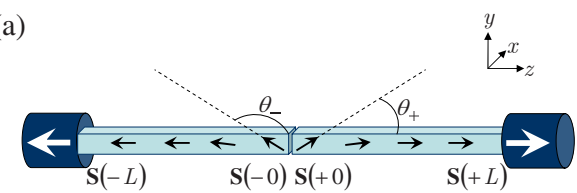

(b)

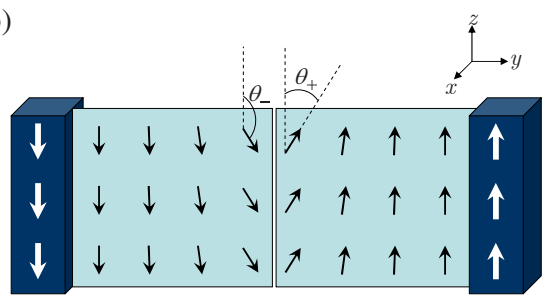

(c)

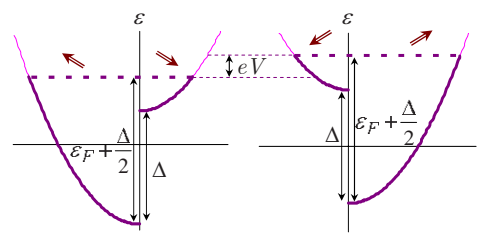

FIG. 1. (Color online) (a) Ferromagnetic wires and (b) ferromagnetic films. In both panels, polarizations of the ends $l=0$ and $l= \pm L$ are $\mathbf{S}( \pm 0)$ and $\mathbf{S}( \pm L)$, respectively. The angle between $\mathbf{S}( \pm 0)$ and axis $z$ is $\theta_{ \pm}$. (c) Band alignment of the majority and minority bands for electrons in the vicinity of the left- and righthand side of a biased ferromagnetic junction in the antiparallel configuration. Direction of spin quantization on either side of the junction is determined by the orientation of $\mathbf{S}( \pm 0)$ of magnetization near the interface. 
term in Eq. (1) with parameters $\xi_{1}>\xi_{2}>0$ describes crystalline anisotropy, whereas the third term is the energy of the ferromagnets in an external magnetic field $\mathbf{B}=B \mathbf{e}_{z}$, where $\mu$ is the magnetic moment per atom. To describe the magnetization of thin wires, we also add a nonlocal dipole-dipole interaction term,

$$
V(l)=\frac{g}{2 w} \int d^{2} \boldsymbol{\rho} d^{2} \boldsymbol{\rho}^{\prime} \frac{2 l^{2}-\left|\boldsymbol{\rho}-\boldsymbol{\rho}^{\prime}\right|^{2}}{\left(\left|\boldsymbol{\rho}-\boldsymbol{\rho}^{\prime}\right|^{2}+l^{2}\right)^{5 / 2}},
$$

where the integration is carried out over the cross section of the wire and $g=\mu^{2} \gamma^{2} / a^{3}$. For $|l| \gg w, V(l)$ decreases as $w^{3} / l^{3}$, and for $|l| \ll w, V(l) \propto \ln (w /|l|)$. For a smooth magnetization texture varying at a length scale longer than the width of a wire, we approximate $V(l) \approx V_{0} w \delta(l)$, where $V_{0}=\int \frac{d l}{w} V(l)$.

The penetration of a polarized electron wave function through the barrier, from one ferromagnetic metal into another, leads to ferromagnetic coupling between them, which is described by the term in Eq. (1),

$$
E^{\prime}=-\frac{t^{\prime} w^{2}}{a^{2}}[\mathbf{S}(+0) \cdot \mathbf{S}(-0)] \text {. }
$$

In the following, we will focus on the magnetization texture formed near the tunnel junction between two ferromagnetic metals with antiparallel polarization $\mathbf{S}( \pm L)= \pm \mathbf{e}_{z}$ fixed by magnetic reservoirs at the distant ends [Figs. 1(a) and $1(\mathrm{~b})]$. Without coupling between ferromagnets $\left(t^{\prime}=0\right)$, this would determine homogeneous magnetizations $\mathbf{S}(l>0)=\mathbf{e}_{z}$ and $\mathbf{S}(l<0)=-\mathbf{e}_{z}$. The exchange interaction across the tunnel barrier may give rise to the formation of canted magnetization texture in the vicinity of the junction with boundary values of spin $\mathbf{S}( \pm 0)$ determined by the interplay between the magnetization rigidity, crystalline anisotropy, Zeeman energy, and ferromagnetic tunneling coupling. In the case when the easy magnetization direction is along $\mathbf{e}_{z}$, we parametrize

$$
\mathbf{S}(l)=\mathbf{e}_{z} \cos [\theta(l)]+\mathbf{e}_{y} \sin [\theta(l)],
$$

with $\theta(-L)=\pi$ and $\theta(L)=0$. Then, the total energy of the interacting ferromagnets takes the form

$$
\begin{aligned}
E= & \frac{J}{2}\left(\int_{-L}^{-0}+\int_{+0}^{L}\right) d l\left\{\left[\theta^{\prime}\right]^{2}+\alpha^{2} \sin ^{2} \theta+2 \alpha^{2} \lambda_{B}[1-\cos \theta]\right\} \\
& -t^{\prime} \cos \left(\theta_{-}-\theta_{+}\right)
\end{aligned}
$$

where $\theta^{\prime}=d \theta / d l$ and $\theta_{ \pm}=\theta( \pm 0)$. Relevant parameters present in Eq. (3) are

$$
\alpha=\sqrt{\frac{w^{2}}{a^{3}} \frac{2\left(\xi_{1}-\xi_{2}\right)+3 V_{0}}{J}}, \quad \lambda_{B}=\frac{B}{B_{0}},
$$

where

$$
B_{0}=\frac{J a^{3} \alpha^{2}}{2 \mu w^{2}} .
$$

For $B>B_{0}$, the polarization antiparallel to the magnetic field is absolutely unstable, leading to a jump in the magnetic domain wall from the junction toward the magnetically biased end of the ferromagnets when $B=B_{0}$. Below we assume that $B_{0}$ is much less than the field required to switch the polarization of the left- or right-hand side lead. Parameters $\alpha$ and $\lambda_{B}$ characterize a typical domain-wall width in a bulk ferromagnet. For example, $\alpha^{-1}$ is the domain width for $B$ $=0$ for $L \alpha \gg 1$. An external magnetic field $\mathbf{B}=B \mathbf{e}_{z}$ makes the domain-wall asymmetric. It compresses the domain-wall width on the side where magnetization is aligned with $\mathbf{B}$ to $\alpha_{+}^{-1}, \alpha_{+}=\alpha \sqrt{1+\lambda_{B}}$, and it stretches the domain-wall width on the side where $\mathbf{S}$ is antiparallel to $\mathbf{B}$ to $\alpha_{-}^{-1}, \alpha_{-}=\alpha \sqrt{1-\lambda_{B}}$.

To minimize the energy in Eq. (3), we employ the following procedure. First, we fix the boundary values $\theta_{ \pm}=\theta( \pm 0)$ and find the optimal form of $\theta(l)$ for given $\theta_{ \pm}$such that the variational derivative of the energy [Eq. (3)] with respect to the vector $\mathbf{S}(l)$ is zero;

$$
\frac{\delta E}{\delta \theta(l)}=0, \quad \text { for } \quad \theta( \pm 0)=\theta_{ \pm} .
$$

This leads to the optimum equation for $\theta(l)$,

$$
\theta^{\prime \prime}=\frac{\alpha^{2}}{2}\left\{\sin [2 \theta]+2 \lambda_{B} \sin \theta\right\} .
$$

The latter shows that $\theta(l>0)[\pi-\theta(l<0)]$ takes its maximal value $\theta_{+}\left[\pi-\theta_{-}\right]$at $l=0$ and that it decreases as $\exp \left(-\alpha_{+} l\right)$ $\left[\exp \left(-\alpha_{-}|l|\right)\right]$ for $|l| \gg \alpha^{-1}$. Also, the differential equation (5) has the first integral $v_{ \pm}(\theta)=-\theta^{\prime}(l)$,

$$
v_{ \pm}(\theta)=\alpha \sqrt{\left(1 \pm \lambda_{B}\right)^{2}-\left[\cos (\theta)+\lambda_{B}\right]^{2}},
$$

where the sign + or - corresponds to $l>0$ or $l<0$. Substituting $v_{ \pm}$into energy in Eq. (3) and changing the integration variable from $l$ to $\theta$, we arrive at

$$
E=J\left[\int_{0}^{\theta_{+}} v_{+}(\theta) d \theta+\int_{\theta_{-}}^{\pi} v_{-}(\theta) d \theta\right]-t^{\prime} \cos \left(\theta_{-}-\theta_{+}\right),
$$

which represents the explicit dependence of energy $E\left(\theta_{+}, \theta_{-}\right)$ on the canting angles on each side of the junction. Minimizing the energy $E\left(\theta_{+}, \theta_{-}\right)$with respect to these angles, we identify possible regimes for the magnetization texture.

First, we consider the magnetization texture in the absence of an external magnetic field, $B=0$. In this case, the texture is symmetric, $\theta(-l)=\pi-\theta(l)$, so that $v_{+}(\theta)=v_{-}(\theta)$ $=\alpha \sin (\theta)$ and

$$
E=2 J \alpha\{1-\cos [\theta(+0)]\}+t^{\prime} \cos [2 \theta(+0)] .
$$

The latter result indicates the existence of a critical value of the coupling constant $t^{\prime}$,

$$
t_{0}=\frac{J \alpha}{2},
$$

such that for $t^{\prime}<t_{0}$ the energy reaches its minimum when $\theta_{+}=\pi-\theta_{-}=0$ and magnetization is homogeneous in each of two ferromagnets, whereas for $t^{\prime}>t_{0}$ the energy minimum corresponds to the presence of magnetization texture in the vicinity of the tunnel junction with

$$
\theta_{+}=\pi-\theta_{-}=\arccos \left(t_{0} / t^{\prime}\right) .
$$




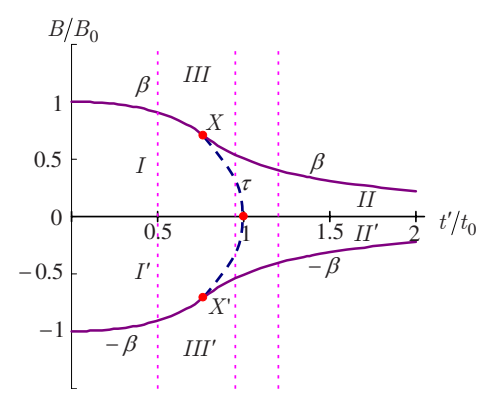

FIG. 2. (Color online) Parametric diagram $t^{\prime}-B$. There are four lines, $\beta$ and $-\beta$ (solid lines), $\tau$ (dashed line), and axis $B=0$ separating magnetization regimes one from another (see the text for details). The lines $\beta$ and $\tau(-\beta$ and $\tau$ ) touch one another at point $\mathrm{X}$ $\left(\mathrm{X}^{\prime}\right)$. The boundary values $\theta_{ \pm}$as a function of the external magnetic field are calculated for $t^{\prime}=0.5 t_{0}, 0.95 t_{0}$, and $1.2 t_{0}$ (thin dashed lines).

In the presence of external magnetic field, the magnetization texture becomes asymmetric. In this case, we determine canting angles $\theta_{ \pm}$numerically from the set of two equations,

$$
\partial_{\theta_{ \pm}} E=J v_{ \pm}\left(\theta_{ \pm}\right)-t^{\prime} \sin \left(\theta_{-}-\theta_{+}\right)=0,
$$

where $v_{ \pm}(\theta)$ [Eq. (6)] includes the magnetic-field dependence.

The results of numerical analysis of Eq. (9) are gathered in the parametric diagram in Fig. 2. Here, the magnetic wall depinning from the tunnel junction is indicated by the critical value of the external magnetic field

$$
B_{\mathrm{c}}\left(t^{\prime}\right)=B_{0} \beta\left(t^{\prime} / t_{0}\right),
$$

which separates the parametric regions I ( $\left.{ }^{\prime}\right)$ and II (II') where magnetization can be antiparallel on the two sides of the junction from region III (III') where magnetization in both ferromagnets is aligned with the external field and the domain wall is pushed away toward the magnetically biased ends of the ferromagnets. For $|B|<B_{\mathrm{c}}\left(t^{\prime}\right)$, the structure of the domain wall depends on the junction transparency $t^{\prime}$. When $t^{\prime}$ is less than critical $t_{\mathrm{c}}(B)$, interval $\mathrm{I}\left(\mathrm{I}^{\prime}\right)$ in Fig. 2,

$$
t_{\mathrm{c}}(B)=t_{0} \tau\left(|B| / B_{0}\right),
$$

the domain wall is atomically sharp and vectors $\mathbf{S}(+0)$ and $\mathbf{S}(-0)$ are antiparallel to one another $\left(\theta_{+}=0\right.$ and $\left.\theta_{-}=\pi\right)$. Sweeping the barrier transparency $t^{\prime}$ across the value $t_{\mathrm{c}}(B)$, parametric interval II (II') in Fig. 2, results in the formation of inhomogeneous magnetization texture in the vicinity of the tunnel junction, whereas the bulk of the ferromagnetic metals is still polarized homogeneously. The values of the canting angles $\theta_{ \pm}$are found from Eq. (9).

A typical calculated dependence of $\theta_{+}$on magnetic field $B$ is illustrated in Fig. 3 for $t^{\prime}=0.5 t_{0}, t^{\prime}=0.95 t_{0}$, and $t^{\prime}=1.2 t_{0}$, respectively, for both $-B \rightarrow B$ and $B \rightarrow-B$ sweeps. The value of $\theta_{-}$can be obtained from Fig. 3 by the transformation

$$
\theta_{-}(B)=\pi-\theta_{+}(-B) .
$$

Figure 3 shows that decreasing the external magnetic field from the value exceeding $B_{\mathrm{c}}\left(t^{\prime}\right)$ results in the movement of the domain wall onto the tunnel junction upon the magnetic- (a)

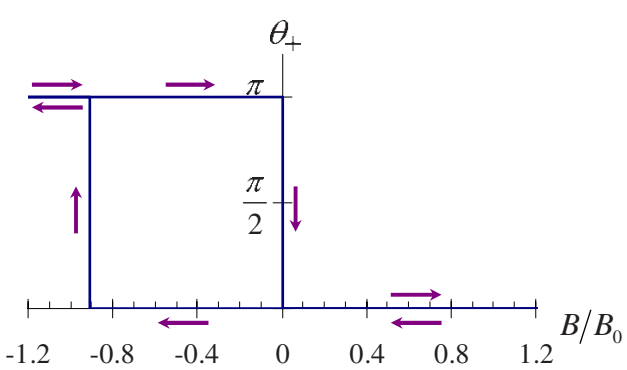

(b)

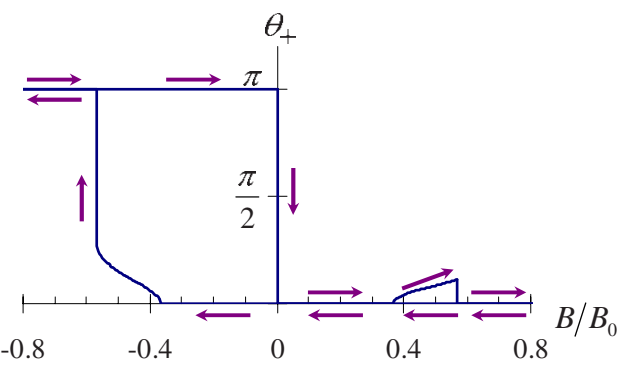

(c)

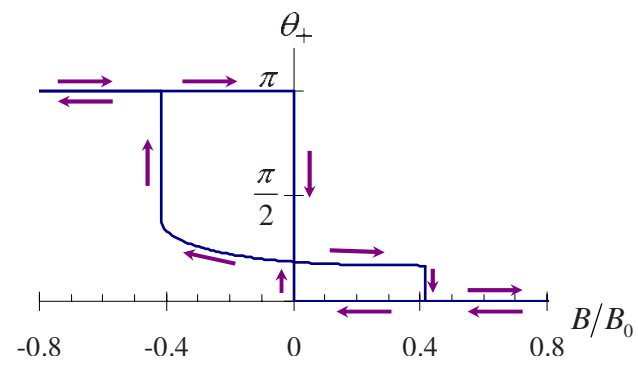

FIG. 3. (Color online) Canting angle $\theta_{+}$as functions of $B$ for $t^{\prime}=0.5 t_{0}$ [panel (a) $], t^{\prime}=0.95 t_{0}$ [panel (b)], and $t^{\prime}=1.2 t_{0}$ [panel (c) $]$.

field sweeps across $B=0$. Further decreasing of the magnetic field leads to the pushing of the domain wall toward the left end of the ferromagnet upon the magnetic-field sweeps across $B=-B_{\mathrm{c}}\left(t^{\prime}\right)$. Reverse sweeping of the external magnetic field results in pushing the domain wall from the left to the right end of the ferromagnetic metal, keeping it near the tunnel junction within the interval $0<B<B_{\mathrm{c}}\left(t^{\prime}\right)$. This difference between $B_{\mathrm{c}}\left(t^{\prime}\right) \rightarrow-B_{\mathrm{c}}\left(t^{\prime}\right)$ and $-B_{\mathrm{c}}\left(t^{\prime}\right) \rightarrow B_{\mathrm{c}}\left(t^{\prime}\right)$ sweeps describes hysteresis in the overall magnetization of two ferromagnets for any values of the coupling $t^{\prime}$.

The formation of canted magnetization in the vicinity of a tunnel junction with a high transparency would affect the magnetoresistance characteristic of such a junction. The latter can be modeled using the tunnel Hamiltonian approach. ${ }^{15-18}$ When the domain-wall width $\alpha^{-1}$ is sufficiently larger than the elastic mean free path, the tunneling Hamiltonian can be written as

$$
H=H_{0}+H_{T}, \quad H_{0}=\sum_{\nu=L, R} \sum_{\mathbf{k}, \sigma} \epsilon_{\sigma}(\mathbf{k}) c_{\nu \mathbf{k} \sigma}^{\dagger} c_{\nu \mathbf{k} \sigma}
$$

where $\epsilon_{\sigma}(\mathbf{k})=\epsilon(\mathbf{k})-\sigma \Delta$. Here $H_{0}$ is the Hamiltonian of the isolated ferromagnetic metals, $H_{T}$ is the tunneling Hamiltonian, $c_{\nu \mathbf{k} \sigma}$ and $c_{\nu \mathbf{k} \sigma}^{\dagger}$ are the annihilation and creation operators of electron propagating in the left $(\nu=L)$ or right $(\nu$ $=R)$ metal with wave vector $\mathbf{k}$ and spin parallel $(\sigma=1 / 2$ or 
$\uparrow)$ or antiparallel $(\sigma=-1 / 2$ or $\downarrow)$ to the ferromagnet polarization $\mathbf{S}( \pm 0)$ [Eq. (2)]. In the following, we will assume that the Fermi momenta for the majority and minority bands are sufficiently larger than $\alpha_{ \pm}$and therefore treat conduction band electrons as three dimensional.

$$
H_{T}=\sum_{\sigma, \sigma^{\prime}} \sum_{\mathbf{k}, \mathbf{k}^{\prime}}\left[t_{\sigma^{\prime} \sigma}\left(\mathbf{k}^{\prime}, \mathbf{k}\right) c_{R \mathbf{k}^{\prime} \sigma^{\prime}}^{\dagger} c_{L \mathbf{k} \sigma}+\text { H.c. }\right],
$$

where the tunneling matrix elements $t_{\sigma^{\prime} \sigma}\left(\mathbf{k}^{\prime}, \mathbf{k}\right)$ describe the transfer of an electron with wave vector $\mathbf{k}$ and spin state $\sigma$ from the left side of the tunnel barrier to the state with $\mathbf{k}^{\prime}$ and $\sigma^{\prime}$ at the right side and the quantization axes for the conduction band electrons are directed along the magnetization vectors $\mathbf{S}( \pm 0)$ [Eq. (2)] which are not necessarily collinear (Fig. 1 ), so that the transitions between the majority or minority band of one metal and majority or minority band of the other are possible even in the nominally antiparallel configuration of $\mathbf{S}( \pm L)$. We consider a model in which electrons tunnel from one metallic ferromagnet to another without spin flipping. In this case the spin dependence of $t_{\sigma^{\prime} \sigma}\left(\mathbf{k}^{\prime}, \mathbf{k}\right)$ is determined by a single parameter, the angle $\theta_{0}=\theta_{-}-\theta_{+}$between the vectors $\mathbf{S}( \pm 0)$,

$$
\begin{aligned}
t_{\sigma \sigma^{\prime}}\left(\mathbf{k}^{\prime}, \mathbf{k}\right)= & \tilde{t}\left|\frac{2 \pi^{2} \hbar^{2} v^{z}(\mathbf{k}) v^{z}\left(\mathbf{k}^{\prime}\right)}{L^{2}}\right|^{1 / 2} \\
& \times\left[\cos \left(\theta_{0} / 2\right) \delta_{\sigma \sigma^{\prime}}+i \sin \left(\theta_{0} / 2\right) \tau_{\sigma \sigma^{\prime}}^{x}\right],
\end{aligned}
$$

where $\tau^{x}$ is the Pauli matrix and $v^{z}(\mathbf{k})$ is a component of electron velocity $\mathbf{v}(\mathbf{k})=\nabla_{\mathbf{k}} \epsilon_{\sigma}(\mathbf{k}) / \hbar$ perpendicular to the interface.

When vectors $\mathbf{S}(+0)$ and $\mathbf{S}(-0)$ are antiparallel (parametric intervals $I$ and $I^{\prime}$ in Fig. 2), electrons can tunnel only from the majority band of one ferromagnetic metal to the minority band of the other ${ }^{10,16,19,20}$ so that the conductance of such a junction is

$$
G_{\uparrow \downarrow}=\frac{2 \pi e^{2}|\vec{t}|^{2}}{\hbar}\left(\frac{2 \pi \hbar}{L}\right)^{2} N_{\uparrow} v_{\uparrow}^{z} N_{\downarrow} v_{\downarrow}^{z},
$$

where $N_{\uparrow}$ and $N_{\downarrow}$ are the densities of states in the majority and minority bands at the Fermi level, and $v_{\uparrow}^{z} / v_{\jmath}^{z}$ are the average value of $\left|v^{z}(\mathbf{k})\right|$ over the majority or minority Fermi surface

$$
v_{\sigma}^{z}=\frac{1}{N_{\sigma}} \sum_{\mathbf{k}}\left|v^{z}(\mathbf{k})\right| \delta\left[\varepsilon_{F}+\sigma \Delta-\epsilon(\mathbf{k})\right] .
$$

When the ends $l= \pm 0$ of the metals have parallel magnetization, i.e., $\theta_{ \pm}=0$ or $\pi$, the conductance is

$$
G_{\uparrow \uparrow}=\frac{2 \pi e^{2}|\widetilde{t}|^{2}}{\hbar}\left(\frac{2 \pi \hbar}{L}\right)^{2} \frac{\left(N_{\uparrow} v_{\uparrow}^{z}\right)^{2}+\left(N_{\downarrow} v_{\downarrow}^{z}\right)^{2}}{2},
$$

which determines the MR ratio

$$
\delta_{0}=\frac{G_{\uparrow \uparrow}-G_{\uparrow \downarrow}}{G_{\uparrow \uparrow}} .
$$

Formation of a canted magnetization texture in the vicinity of a junction with angle $\theta_{0}=\theta_{-}-\theta_{+}$between magnetiza- (a)
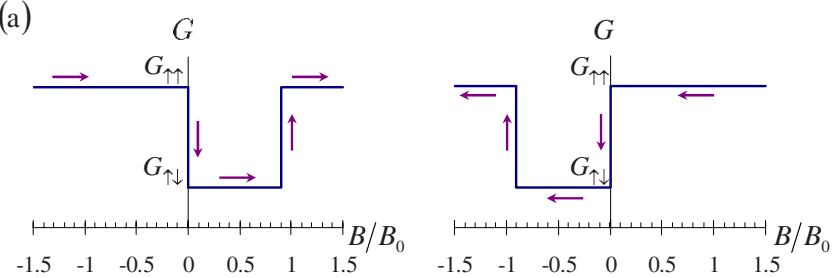

(b)
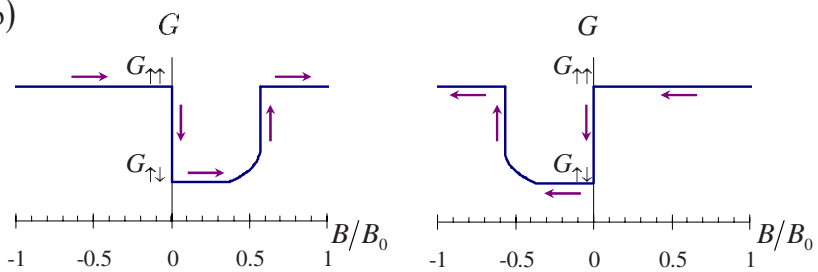

(c)
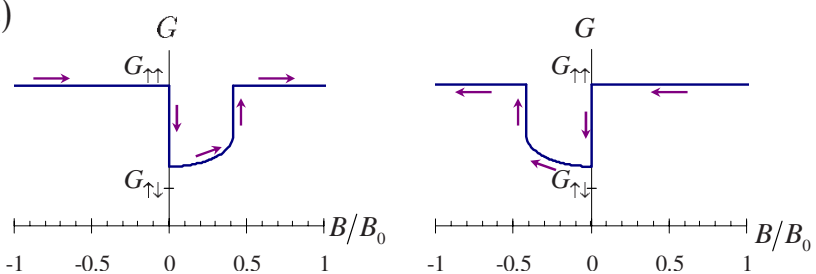

FIG. 4. (Color online) Magnetoresistance for positive (left column) and negative (right column) magnetic-field sweeps showing the effect of canted magnetization texture on the form of hysteresis in the magnetic tunnel junction for (a) $t^{\prime}=0.5 t_{0}$, (b) $t^{\prime}=0.95 t_{0}$, and (c) $t^{\prime}=1.2 t_{0}$.

tion directions of the opposite sides of the tunnel barrier produces conductance $G\left(\theta_{0}\right)$ and the reduced observable MR ratio, $\delta$,

$$
\begin{aligned}
G\left(\theta_{0}\right) & =G_{\uparrow \uparrow} \cos ^{2}\left(\theta_{0} / 2\right)+G_{\uparrow \downarrow} \sin ^{2}\left(\theta_{0} / 2\right), \\
\delta & =\frac{G_{\uparrow \uparrow}-G\left(\theta_{0}\right)}{G_{\uparrow \uparrow}}=\delta_{0} \sin ^{2}\left(\theta_{0} / 2\right) .
\end{aligned}
$$

The results of calculations for the conductance in MR devices with various junction transparencies (and positive and negative magnetic-field sweeps in the magnetoresistance) are gathered in Fig. 4. The conductance dip at small magnetic fields indicates the regime when a ferromagnetic domain wall is pinned to the tunnel junction. The difference between positive and negative sweeps (left- and right-hand side columns) is a manifestation of the hysteresis in the overall magnetization of two ferromagnetic metals. In both cases, a domain wall falls onto the tunnel junction upon the magnetic-field sweep across $B=0$ and remains pinned to it until the field exceeds the value $B_{\mathrm{c}}\left(t^{\prime}\right)\left[-B_{\mathrm{c}}\left(t^{\prime}\right)\right]$ separating parametric regimes I or II and III ( $\mathrm{I}^{\prime}$ or II' and III') in Fig. 2. The detailed structure of the hysteresis loop depends on whether the magnetization texture is formed near the junction or not, depending on the value of the ferromagnetic coupling across the barrier. For a small coupling, in Fig. 4(a), the jumps between parallel and antiparallel polarizations in the vicinity of the tunnel junction give rise to jumps in the conductance between $G_{\uparrow \uparrow}$ and a flat conductance minimum equal to $G_{\uparrow \downarrow}$. By increasing the coupling toward the critical 
value $t_{0}$ (determined by the interplay between crystalline anisotropy and magnetization rigidity in ferromagnet), an interval of magnetic fields appears where the conductance [Eq. (12)] increases continuously due to the formation of a canted magnetization texture and its change by a magnetic field [Fig. 4(b)]. For the coupling above, a critical value $t_{0}$ [Fig. 4(c)] the minimum of the conductance exceeds $G_{\uparrow \downarrow}$ even in the absence of a magnetic field, indicating the formation of a broad texture, which also suggests a reduction in MR ratio in a high-transparency junction.

The authors thank G. Bauer and E. McCann for useful discussions. This project has been funded by EU STREP DynaMax and ESF CRP "Spincurrent."
${ }^{1}$ G. A. Prinz, Phys. Today 48(4), 58 (1995); Science 282, 1660 (1998), and references therein.

${ }^{2}$ A. Brataas, G. E. W. Bauer, and P. J. Kelly, Phys. Rep. 427, 157 (2006).

${ }^{3}$ M. Julliere, Phys. Lett. 54A, 225 (1975).

${ }^{4}$ J. S. Moodera, T. H. Kim, C. Tanaka, and C. H. de Groot, Philos. Mag. B 80, 195 (2000).

${ }^{5}$ G. Usaj and H. U. Baranger, Phys. Rev. B 63, 184418 (2001).

${ }^{6}$ A. Gerber, A. Milner, B. Groisman, M. Karpovsky, A. Gladkikh, and A. Sulpice, Phys. Rev. B 55, 6446 (1997).

${ }^{7}$ M. N. Baibich, J. M. Broto, A. Fert, F. Nguyen Van Dau, F. Petroff, P. Etienne, G. Creuzet, A. Friederich, and J. Chazelas, Phys. Rev. Lett. 61, 2472 (1988).

${ }^{8}$ G. Binasch, P. Grünberg, F. Saurenbach, and W. Zinn, Phys. Rev. B 39, 4828(R) (1989).

${ }^{9}$ W. P. Pratt, Jr., S.-F. Lee, J. M. Slaughter, R. Loloee, P. A. Schroeder, and J. Bass, Phys. Rev. Lett. 66, 3060 (1991); S. F. Lee, W. P. Pratt, Jr., R. Loloee, P. A. Schroeder, and J. Bass, Phys. Rev. B 46, 548 (1992).
${ }^{10}$ Y. Sakuraba, M. Hattori, M. Oogane, Y. Ando, H. Kato, A. Sakuma, T. Miyazaki, and H. Kubota, Appl. Phys. Lett. 88, 192508 (2006).

${ }^{11}$ J. S. Moodera, L. R. Kinder, T. M. Wong, and R. Meservey, Phys. Rev. Lett. 74, 3273 (1995).

${ }^{12}$ I. Žutić, J. Fabian, and S. Das Sarma, Rev. Mod. Phys. 76, 323 (2004).

${ }^{13}$ J. Mathon and A. Umerski, Phys. Rev. B 63, 220403(R) (2001).

${ }^{14}$ Y. Li, B. Z. Li, W. S. Zhang, and D. S. Dai, Phys. Rev. B 57, 1079 (1998).

${ }^{15}$ G. D. Mahan, Many-Particle Physics (Plenum, New York, 1981).

${ }^{16}$ E. McCann and V. I. Falko, Phys. Rev. B 66, 134424 (2002).

${ }^{17}$ F. S. Nogueira and K.-H. Bennemann, Europhys. Lett. 67, 620 (2004).

${ }^{18}$ A. M. Bratkovsky, Phys. Rev. B 56, 2344 (1997).

${ }^{19}$ J. Shi, K. Pettit, E. Kita, S. S. P. Parkin, R. Nakatani, and M. B. Salamon, Phys. Rev. B 54, 15273 (1996).

${ }^{20}$ E. Yu. Tsymbal, D. G. Pettifor, J. Shi, and M. B. Salamon, Phys. Rev. B 59, 8371 (1999). 